\title{
Low expression of BMPRIB indicates poor prognosis of breast cancer and is insensitive to taxane-anthracycline chemotherapy
}

\author{
Kun Dai ${ }^{1}$, Fengxia Qin ${ }^{1}$, Huikun Zhang ${ }^{1}$, Xiaoli Liu ${ }^{2}$, Caixia Guo ${ }^{3}$, Ming Zhang ${ }^{4}$, Feng Gu $^{1}$, \\ Li Fu' ${ }^{1}$, Yongjie $\mathrm{Ma}^{2}$ \\ ${ }^{1}$ Department of Breast Cancer Pathology and Research Laboratory, Key Laboratory of Breast Cancer Prevention and Therapy \\ (Ministry of Education), Tianjin Medical University Cancer Institute and Hospital, National Clinical Research Center for \\ Cancer, Tianjin, China \\ ${ }^{2}$ Department of Tumor Cell Biology, Key Laboratory of Cancer Prevention and Therapy of Tianjin, Tianjin Medical University \\ Cancer Institute and Hospital, National Clinical Research Center for Cancer, Tianjin, China \\ ${ }^{3}$ CAS Key Laboratory of Genomic and Precision Medicine, Beijing Institute of Genomics, Chinese Academy of Sciences, \\ Beijing, China \\ ${ }^{4}$ Department of Epidemiology and Biostatistics, University of Georgia, Athens, GA, U.S.A \\ Correspondence to: Yongjie Ma, e-mail: mayongjie@tjmuch.com \\ Feng Gu, e-mail: fenggumayo@163.com \\ Li Fu, e-mail: fulijyb@hotmail.com
}

Keywords: BMPRIB, breast cancer, bone metastasis, TE chemotherapy, prognosis

Received: September 10, $2015 \quad$ Accepted: November 26, 2015

Published: December 14, 2015

\section{ABSTRACT}

Bone morphogenetic protein receptor type IB (BMPRIB) is one osteogenesis factor, which function in breast cancer has been rarely explored until recently. In the clinical study presented here, involving a cohort of 368 invasive ductal carcinoma (IDC) patients, we identified that patients with low expression of BMPRIB exhibited poor prognosis, especially in the luminal B subtype. We also provided the first piece of evidence that low level of BMPRIB was a promoting factor for breast cancer patients to develop bone metastasis, but not lung, liver or brain. The first of its kind, we reported that patients with high expression of BMPRIB exhibited favorable prognosis by a retrospective analysis consisting of 168 patients treated with TE (taxane and anthracycline) regimens. And the patients with high expression of BMPRIB were more sensitive to TE regimens in the detection of 32 paired pre-neoadjuvant and post-neoadjuvant specimens. Overall, our study concluded that low expression of BMPRIB indicated poor prognosis of breast cancer and was insensitive to taxane-anthracycline chemotherapy. Our findings also lay a foundation to help clinicians improve identification of patients for TE regimens by BMPRIB in the era of precision medicine.

\section{INTRODUCTION}

Breast cancer is a common cancer in women and a major cause of cancer death among women of all races and populations, due to the development of secondary tumors in vital organs [1]. First metastatic lesions are found at the highest frequency in bone (83\%) whereas liver and lung are usually affected to a lesser extent (27\%) [2-3].

Bone morphogenetic protein receptor type IB (BMPRIB) as a member of BMPRs, could lead to the activation of intracellular signaling pathways and inhibited cell proliferation, migration, and invasiveness [4-7]. It has been reported that BMPRIB expression was higher in the normal, benign specimens and well-differentiated tumors compared with poorly-differentiated tumors in human prostate cancer, glioma, and ovarian cancer [8-12].

Reports of BMPRIB in breast cancer are few and contradictory $[5,13]$. It was reported high expression of BMPRIB indicated a poor prognosis in estrogen receptorpositive breast cancer patients [13]. However, Bokobza et al.'s study presented an opposite conclusion in prognosis [5]. Such confused reports were probably due to small samples or unscientific division. Until present, there is still no comprehensive description which is capable of pointing out the accurate relationship between BMPRIB and breast cancer, particularly distant metastasis. 
In our present clinicopathologic study, we presented immunohistochemistry analysis of BMPRIB expression in a large population of 368 cases of invasive ductal carcinoma (IDC), which covered all molecular subtypes. We confirmed that low expression of BMPRIB predicted poor prognosis in IDC patients. In addition, we found that IDC patients with low expression of BMPRIB exhibited bone-specific metastasis, but not lung, liver or brain. Furthermore, we reported firstly that high level of BMPRIB expression predicted a favorable prognosis in patients treated with TE (taxane and anthracycline) regimens. Patients with high expression of BMPRIB were more sensitive to TE regimens which was validated by detecting 32 paired pre-neoadjuvant and post-neoadjuvant specimens. Overall, our findings demonstrated that low expression of BMPRIB indicated poor prognosis of breast cancer and was insensitive to taxane-anthracycline chemotherapy.

\section{RESULTS}

\section{Low expression of BMPRIB promoted breast cancer progression}

Expression of BMPRIB was detected in 52 cases of non-neoplastic tissues adjacent to tumor, 40 cases of DCIS and 368 cases of IDC. The immunohistochemical staining of BMPRIB was assessed and the intensity of staining was shown in representative images as Figure 1A. In breast

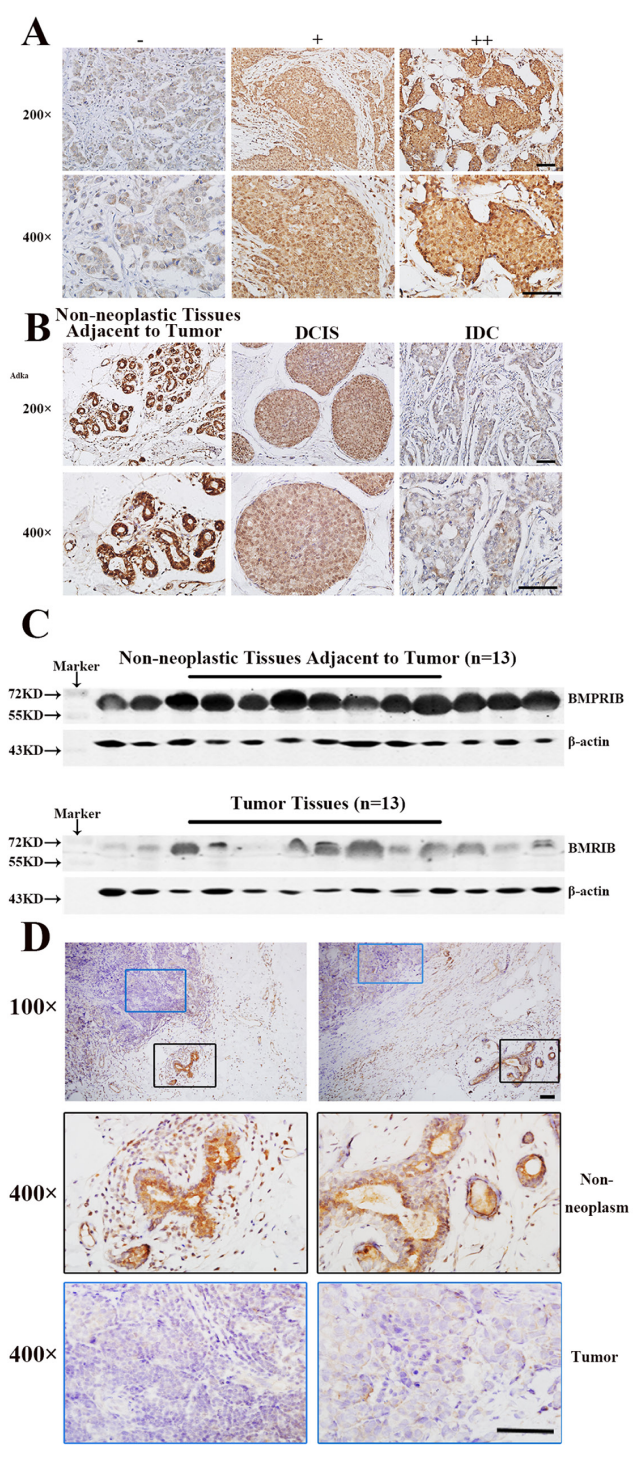

Figure 1: Low expression of BMPRIB promoted breast cancer progression. A. Varying degree staining intensity of BMPRIB protein in invasive ductal carcinoma specimens: (-): no or low staining; $(+)$ : moderate staining; $(++)$ : strong staining. B. Immunohistochemistry of BMPRIB in clinical specimens of non-neoplastic breast tissues adjacent to tumor, ductal carcinoma in situ (DCIS) and invasive ductal carcinoma (IDC) (magnification 200× and 400×). C. Western blot analysis of BMPRIB expression in breast tumor specimens $(\mathrm{n}=13)$ and non-neoplastic breast tissues adjacent to tumor $(n=13)$. $\beta$-actin was used as a loading control. D. BMPRIB expression was detected in two typical sections (left part and right part) respectively, both of which contained non-neoplastic tissues and tumor tissues. Blue rectangle represented tumors and black rectangle represented non-neoplastic tissues adjacent to tumor. $(\mathrm{Bar}=100 \mu \mathrm{m})$ 
Table 1: BMPRIB expression in different breast tissue specimens

\begin{tabular}{|c|c|c|c|c|c|}
\hline \multirow[t]{2}{*}{ Histological type } & \multirow[t]{2}{*}{$\mathbf{n}$} & \multicolumn{2}{|c|}{ BMPRIB score, n (\%) } & \multirow[t]{2}{*}{$\boldsymbol{r}_{s}$} & \multirow[t]{2}{*}{$P$ value } \\
\hline & & Low (0-140) & High (141-200) & & \\
\hline Non-neoplastic Tissues & 52 & $14(26.9)$ & $38(73.1)$ & & \\
\hline DCIS & 40 & $22(55.0)$ & $18(45.0)$ & -0.184 & $<0.001 * *$ \\
\hline IDC & 368 & $222(60.3)$ & $146(39.7)$ & & \\
\hline
\end{tabular}

Non-neoplastic Tissues: Non-neoplastic Tissues Adjacent to Tumor

DCIS: ductal carcinoma in situ

** $P$ value was calculated by Spearman's Rank-Correlation test.

tissues, BMPRIB was mainly located in the cytoplasm of epithelial cells of the mammary gland ducts. We found the expression of BMPRIB was gradually down-regulated from non-neoplastic tissues adjacent to tumor, to DCIS and to IDC (Figure 1B). 26.9\% (14/52) of non-neoplastic tissues adjacent to tumor, $55.0 \%(22 / 40)$ of DCIS and $60.3 \%(222 / 368)$ of IDC tissue specimens showed low expression of BMPRIB $\left(r_{s}=-0.184, P<0.001\right)$ (Table 1). Furthermore, Western blot analyses were employed to show the protein expression of BMPRIB in frozen IDC specimens (13 cases) and non-neoplastic breast specimens (13 cases), respectively. We confirmed that expression of BMPRIB was lower in IDC specimens compared with non-neoplastic breast specimens (Figure 1C). We also examined BMPRIB expression in paraffin sections and each section contained both non-neoplasm and tumor. The typical immunohistochemistry images of BMPRIB expression in two cases were shown in Figure 1D.

BMPRIB expression was negatively correlated with tumor size $\left(r_{s}=-0.190, P<0.001\right)$, cTNM stage $\left(r_{s}=\right.$ -0.126, $P=0.016)$, lymph node metastasis $\left(r_{s}=-0.202, P\right.$ $<0.001)$ and distant metastasis $\left(r_{s}=-0.148, P=0.004\right)$ but positively associated with the expression of PR $\left(r_{s}=0.210\right.$, $P<0.001)$ of breast cancer. No significant associations were identified between the expression of BMPRIB and patients' age $\left(r_{s}=-0.056, P=0.286\right)$, histological grade $(r$ $=0.038, P=0.472)$, ER $\left(r_{s}=0.064, P=0.223\right)$, or HER2 $\left(r_{s}=0.016, P=0.758\right)($ Table 2$)$.

\section{Low expression of BMPRIB in IDC patients indicated worse prognosis}

In order to explore the role of BMPRIB in breast cancer prognosis, we analyzed 357 IDC patients with complete clinical follow-up. We found BMPRIB expression in patients with metastasis, recurrence or death within 5 years (H score: 60.0 to 180.0, median: 100.0) was lower than those who were disease-free over 5 years $(\mathrm{H}$ score: 80.0 to 200.0 , median: 130.0$)(P<0.001$, Figure $2 \mathrm{~A})$. Cases with low BMPRIB expression were $87.5 \%$ (42/48) and 51.4\% (74/144) in metastasis, recurrence or death within 5 years group and disease-free over 5 years group $(P<0.001)$ (Figure 2B). Both PFS and OS in IDC patients with low expression of BMPRIB were shorter than that of patients with high expression of BMPRIB (Figure 2C, 2D).

Kaplan-Meier analysis of triple negative subtype (56 cases) showed that the expression of BMPRIB was not correlated with PFS $(P=0.124$, Figure $2 \mathrm{E})$ or OS $(P=$ 0.315 , Figure $2 \mathrm{~F}$ ). Then we investigated the correlation between BMPRIB and prognosis in non-triple negative subtype patients $(\mathrm{n}=301)$. Kaplan-Meier analysis showed that low expression of BMPRIB indicated shorter PFS $(P=0.003$, Figure $2 \mathrm{G})$ and $\mathrm{OS}(P=0.015$, Figure $2 \mathrm{H})$. In the following, we divided non-triple negative cases into two groups: luminal subtype (278 cases) and HER2-overexpression subtype (23 cases). We found no correlation between BMPRIB and PFS $(P=0.228$, Figure $2 \mathrm{~K})$ or OS $(P=0.225$, Figure 2L) in HER2-overexpression subtype. However, Kaplan-Meier analysis showed that low expression of BMPRIB indicated shorter PFS $(P=$ 0.005 , Figure 2I) and OS $(P=0.039$, Figure $2 \mathrm{~J})$ in luminal subtype, especially in luminal B subtype (PFS: $P=0.008$; OS: $P=0.036$; Figure 3 )

\section{Low level of BMPRIB was a promoting factor for breast cancer patients to develop bone metastasis}

In the present study, we found a negative correlation between expression of BMPRIB and bone metastasis in 357 IDC patients $\left(r_{s}=-0.119, P=0.024\right)$. No correlations were identified between BMPRIB expression and distant metastasis to other organs, such as lung $\left(r_{s}=-0.056, P=\right.$ $0.289)$, liver $\left(r_{s}=-0.036, P=0.500\right)$ and brain $\left(r_{s}=-0.048\right.$, $P=0.364$, only 5 cases, data not shown) (Table 3 ).

To further investigate the relationship between BMPRIB expression and breast cancer bone metastasis, 357 IDC patients were divided into 2 groups: breast cancer with bone metastasis $(n=39)$ and without bone metastasis $(\mathrm{n}=318)$. Expression of BMPRIB of breast cancer patients with bone metastasis was lower than that without bone metastasis (Figure 4A). The median H score of BMPRIB (110.0) of patients with bone metastasis was lower than that (130.0) without bone metastasis $(P=0.008$, Figure $4 \mathrm{~B})$. In triple negative patients, BMPRIB expression in the group with bone metastasis (median, 100.0) was similar to that without bone metastasis (median, 120.0) $(P=0.185$, 
Table 2: BMPRIB expression and pathological features of IDC

\begin{tabular}{|c|c|c|c|c|c|}
\hline \multirow[t]{2}{*}{ Pathological features } & \multirow[t]{2}{*}{ n } & \multicolumn{2}{|c|}{ BMPRIB score, n (\%) } & \multirow[t]{2}{*}{$\boldsymbol{r}_{s}$} & \multirow[t]{2}{*}{$P$ value } \\
\hline & & Low (0-140) & High (141-200) & & \\
\hline Age, year & & & & -0.056 & 0.286 \\
\hline$<50$ & 189 & $109(57.7)$ & $80(42.3)$ & & \\
\hline$\geq 50$ & 179 & $113(63.1)$ & $66(36.9)$ & & \\
\hline Tumor size, cm & & & & -0.190 & $<0.001 * *$ \\
\hline$\leq 2$ & 79 & $34(43.0)$ & $45(57.0)$ & & \\
\hline $2-5$ & 230 & $145(63.0)$ & $85(37.0)$ & & \\
\hline$>5$ & 59 & $43(72.9)$ & $16(27.1)$ & & \\
\hline cTNM stage & & & & -0.126 & $0.016^{*}$ \\
\hline I & 60 & $28(46.7)$ & $32(53.3)$ & & \\
\hline II & 237 & $146(61.6)$ & $91(38.4)$ & & \\
\hline III-IV & 69 & $47(68.1)$ & $22(31.9)$ & & \\
\hline Histological grade $\neq$ & & & & 0.038 & 0.472 \\
\hline I & 10 & $5(50.0)$ & $5(50.0)$ & & \\
\hline II & 272 & $164(60.3)$ & $108(39.7)$ & & \\
\hline III & 64 & $37(57.8)$ & $27(42.2)$ & & \\
\hline LN metastasis & & & & -0.202 & $<0.001 * *$ \\
\hline NO & 142 & $68(47.9)$ & $74(52.1)$ & & \\
\hline YES & 226 & $154(68.1)$ & $72(31.9)$ & & \\
\hline Distant metastasis & & & & -0.148 & $0.004 * *$ \\
\hline NO & 317 & $182(57.4)$ & $135(42.6)$ & & \\
\hline YES & 51 & $40(78.4)$ & $11(21.6)$ & & \\
\hline Estrogen receptor & & & & 0.064 & 0.223 \\
\hline Negative & 122 & $79(64.8)$ & $43(35.2)$ & & \\
\hline Positive & 246 & $143(58.1)$ & $103(41.9)$ & & \\
\hline Progesterone receptor & & & & 0.210 & $<0.001 * *$ \\
\hline Negative & 109 & $83(76.1)$ & $26(23.9)$ & & \\
\hline Positive & 259 & $139(53.7)$ & $120(46.3)$ & & \\
\hline HER2/neu & & & & 0.016 & 0.758 \\
\hline Negative & 291 & $180(61.9)$ & $111(38.1)$ & & \\
\hline Positive & 77 & $42(54.5)$ & $35(45.5)$ & & \\
\hline
\end{tabular}

キSome missing data.

LN metastasis: lymph node metastasis

$P$ values were calculated by Spearman's Rank-Correlation test.

Figure 4C). While in non-triple negative subtype patients, Mann-Whitney U analysis showed that patients with bone metastasis (median, 120.0) showed lower median H score of BMPRIB compared with those without bone metastasis (median, 130.0) $(P=0.026$, Figure 4D). Furthermore, in luminal subtype, we found lower expression of BMPRIB in patients with bone metastasis compared with those without bone metastasis $(P=0.027$, Figure 4E). We did not analyze relationship between BMPRIB expression and 
A

\section{.}

C
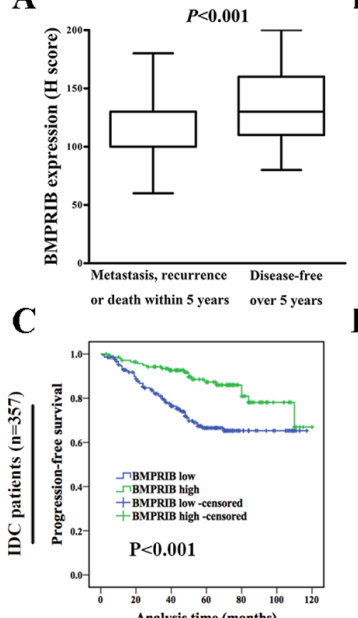

$\mathbf{E}$

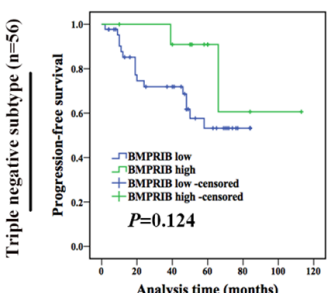

G
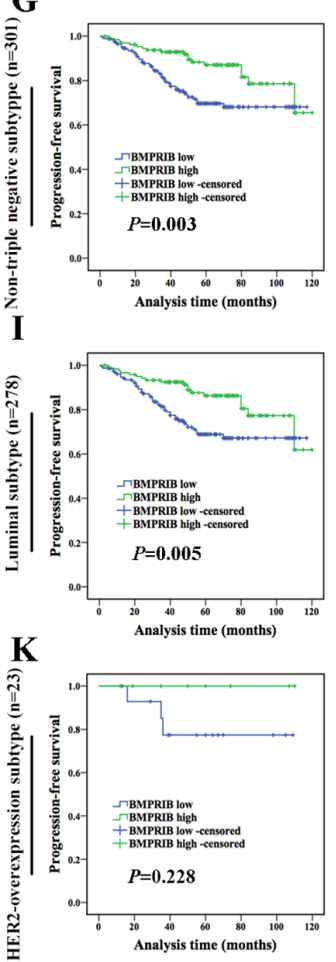

B
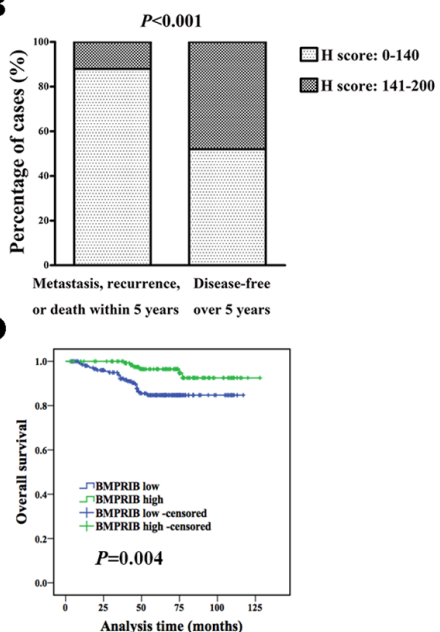

F

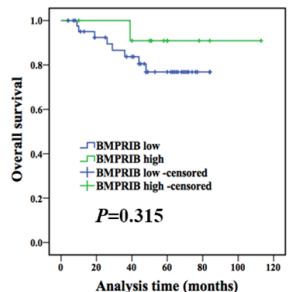

H
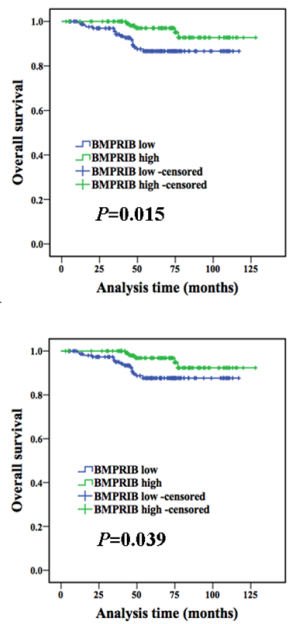

$\mathbf{L}$

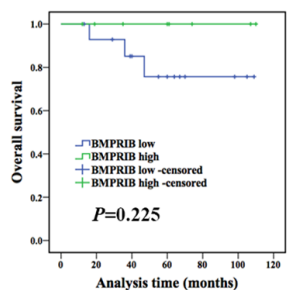

Figure 2: Low expression of BMPRIB in IDC patients indicated worse prognosis. A. BMPRIB expression in patients who developed metastasis, recurrence or death within 5 years was lower than that in those who were disease-free over 5 years (Mann-Whitney U test, $P<0.001)$. B. Percentage of cases with low and high expression of BMPRIB with regard to patients' prognosis. $87.5 \%(42 / 48)$ of patients who developed metastasis, recurrence or death within 5 years showed lower expression of BMPRIB, while 50.7\% (74/144) of patients were disease-free over 5 years $\left(\chi^{2}\right.$ test, $\left.P<0.001\right)$. C and $\mathbf{D}$. Progression-free survival (PFS) and overall survival (OS) curves of IDC patients $(\mathrm{n}=357)$ with BMPRIB expression, respectively (log-rank test). $\mathbf{E}$ and $\mathbf{F}$. PFS and OS curves of triple negative subtype patients $(\mathrm{n}=56)$ with BMPRIB expression, respectively. $\mathbf{G}$ and $\mathbf{H}$. PFS and OS curves of non-triple negative subtype patients $(\mathrm{n}=301)$ with BMPRIB expression, respectively. I and J. PFS and OS curves of luminal subtype patients $(\mathrm{n}=278)$ with BMPRIB expression, respectively. $\mathbf{K}$ and $\mathbf{L}$. PFS and OS curves of HER2-overexpression subtype patients $(\mathrm{n}=23)$ with BMPRIB expression, respectively. 
breast cancer bone metastasis in HER2-overexpression subtype due to only one case.

Next, we analyzed the interval time from diagnosis of breast cancer to bone metastasis and from diagnosis of bone metastasis to the follow-up ending time respectively in the 39 IDC patients with bone metastasis. IDC patients with low BMPRIB expression exhibited a trend for shorter time (median 30.0 months) to develop bone metastasis than those (median 60.0 months) with high expression $(P$
$=0.011$, Figure 4F). Furthermore, the interval time from diagnosis of bone metastasis to the follow-up ending time exhibited the same trend. Low BMPRIB expression has the potential for a reduced survival compared with those with high expression, and the median interval time were 13.0 months and 25.0 months, respectively $(P=0.016$, Figure 4G). Above results indicated that high expression of BMPRIB predicted a favorable outcome in breast cancer with bone metastasis.
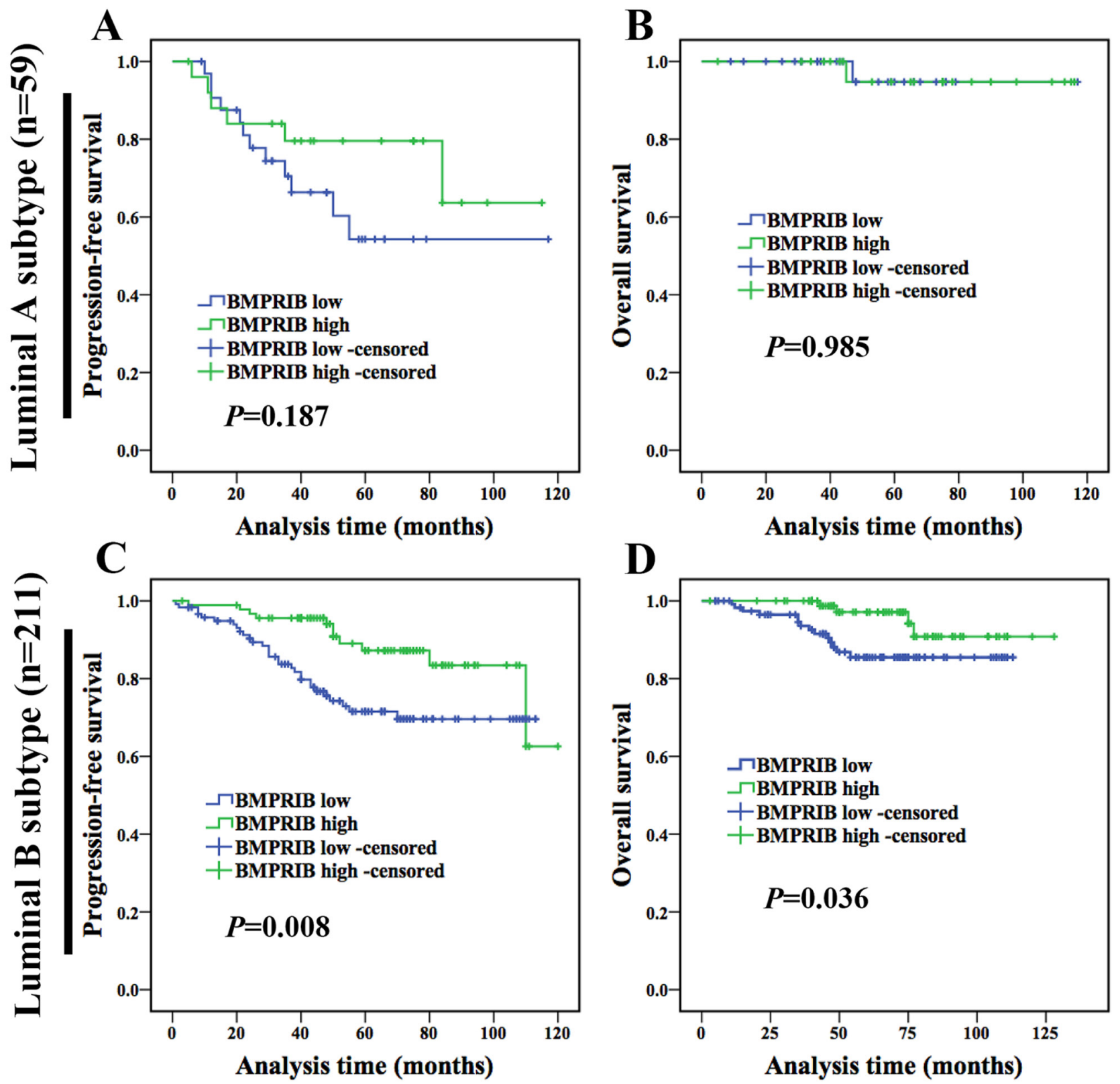

Figure 3: Low expression of BMPRIB in luminal B subtype patients indicated worse prognosis. A and B were PFS and OS curves of luminal A subtype patients $(n=59)$ with BMPRIB expression, respectively. $\mathbf{C}$ and $\mathbf{D}$ were PFS and OS curves of luminal B subtype patients $(\mathrm{n}=211)$ with BMPRIB expression, respectively. 
Table 3: Relationship between BMPRIB expression and distant metastasis in invasive breast cancer

\begin{tabular}{|c|c|c|c|c|c|}
\hline \multirow[t]{2}{*}{ Distant metastasis } & \multirow[t]{2}{*}{$\mathbf{n}$} & \multicolumn{2}{|c|}{ BMPRIB score, n (\%) } & \multirow[t]{2}{*}{$\boldsymbol{r}_{s}$} & \multirow[t]{2}{*}{$P$ value } \\
\hline & & Low $(0-140)$ & High (141-200) & & \\
\hline Bone metastasis & & & & -0.119 & $0.024 *$ \\
\hline NO & 318 & $185(58.2)$ & $133(41.8)$ & & \\
\hline Yes & 39 & $30(76.9)$ & $9(23.1)$ & & \\
\hline Lung metastasis & & & & -0.056 & 0.289 \\
\hline No & 345 & $206(59.7)$ & $139(40.3)$ & & \\
\hline Yes & 12 & $9(75.0)$ & $3(25.0)$ & & \\
\hline Liver metastasis & & & & -0.036 & 0.500 \\
\hline No & 344 & $206(59.9)$ & $138(40.1)$ & & \\
\hline Yes & 13 & $9(69.2)$ & $4(30.8)$ & & \\
\hline
\end{tabular}

$P$ value was calculated by Spearman's Rank-Correlation test.

\section{High expression of BMPRIB indicated a favorable prognosis of breast cancer patients treated with TE-based regimens}

It was reported that docetaxel treatment was associated with expression of BMPRIB. Docetaxel (analogs of taxane) could reduce the expression of $\beta 1$-integrin and then activated BMP signaling by upregulating SMAD1/5/8 and p38 leading to an increased expression of BMPRIB [14-16]. In the following experiments, we examined the relationship of BMPRIB and taxane combined anthracycline (TE) based therapies.

We analyzed the BMPRIB expression with patient prognosis using the immunohistochemcal system in conventional TE-based regimens. Among 357 cases, 168 (47.1\%) patients received TE-based chemotherapy and BMPRIB expression in patients (29 cases) who developed metastasis, recurrence or death within 5 years was lower (median, 100.0) than those (67 cases) who were diseasefree over 5 years (median, 130.0) $(P=0.002$, Figure $5 \mathrm{~A})$. Percentages of cases with low BMPRIB expression were $96.6 \%(28 / 29)$ and $59.7 \%(40 / 67)$ in patients who developed metastasis, recurrence or death within 5 years and patients who were disease-free over 5 years, respectively $\left(\chi^{2}=13.303, P<0.001\right)$ (Figure 5B).

Kaplan-Meier analysis showed that low expression of BMPRIB indicated shorter PFS $(P=0.015$, Figure 5C) and $\mathrm{OS}(P=0.023$, Figure 5D) in patients who received TE-based therapies and followed up with a median of 60 months (5-120 months). We found no correlation between the expression of BMPRIB and OS $(P=0.072$, Figure 5F) in patients (189 cases) who received other chemotherapy, although low expression of BMPRIB indicated a shorter $\mathrm{PFS}(\mathrm{P}=0.030$, Figure 5E). It demonstrated that high expression of BMPRIB indicated a favorable prognosis in patients who received TE-based therapies. Among 168 TE-based cases, we assessed the prognosis in different molecular subtypes. We found no correlation between BMPRIB and OS in luminal subtype (123 cases, $P=$ $0.129)$, triple negative subtype (27 cases, $P=0.342$ ) and HER2-overexpression subtype (15 cases, $P=0.515$ ) (data not shown).

\section{Patients with low expression of BMPRIB were insensitive to $\mathrm{TE}$ regimens}

We also randomly selected another cohort of patients (32 cases) hospitalized during 2005 to 2009 and they were diagnosed with invasive breast cancer using core needle biopsy and then treated with TE combined chemotherapy before surgery. We divided 32 patients into positive pathological response group and negative pathological response group, on the basis of postoperative pathology. We found BMPRIB expression was higher (median, 100) in positive pathological response group (20 cases) than that (median, 80) of negative pathological response group (12 cases) in core needle biopsy specimens before TE treatment (Figure 6A, 6B; $P=0.040$ ).

We found the expression of BMPRIB increased after TE neoadjuvant chemotherapy by using 32 paired specimens of pre- and post-neoadjuvant chemotherapy. Mann-Whitney $\mathrm{U}$ analysis showed that the median $\mathrm{H}$ score of BMPRIB in pre-neoadjuvant chemotherapy specimens (median: 100.0) was lower than that in post-neoadjuvant chemotherapy specimens (median: 130.0) $(P<0.001$, Figure 7A). Percentages of cases with low BMPRIB expression were $87.5 \%(28 / 32)$ and $59.4 \%(19 / 32)$ in groups of pre-neoadjuvant chemotherapy specimens and the post-neoadjuvant chemotherapy specimens, respectively $(P=0.007$, Table 4$)$. In positive pathological response group, $80.0 \%(16 / 20)$ of pre-neoadjuvant chemotherapy specimens showed low expression of BMPRIB, which was higher than that $(50.0 \%, 10 / 20)$ of the post-neoadjuvant chemotherapy specimens $(P=0.034$, 


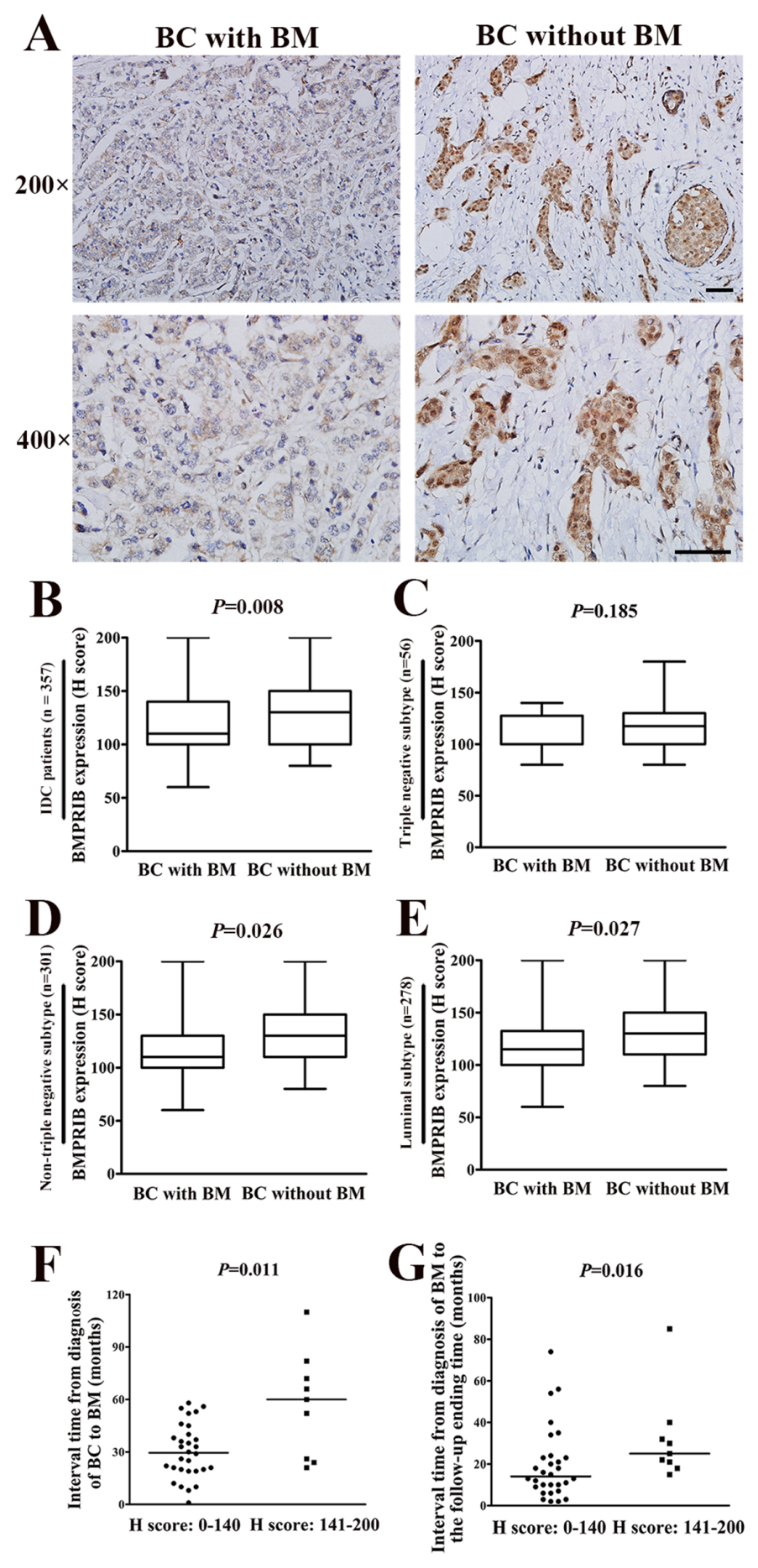

Figure 4: Low level of BMPRIB was a promoting factor for breast cancer (BC) patients to develop bone metastasis (BM). A. Representative immunohistochemical images of BMPRIB expression in primary tumor specimens of BC with BM and BC without BM, respectively (magnification $200 \times$ and $400 \times)$. $(\mathrm{Bar}=100 \mu \mathrm{m}) \mathrm{B}$. BMPRIB expression in BC patients who developed BM was lower than that in those patients without BM $(P=0.008)$. C. Comparison of BMPRIB expression in BC with BM and BC without BM in triple negative subtype IDC patients $(P=0.185)$. D. Comparison of BMPRIB expression in $\mathrm{BC}$ with $\mathrm{BM}$ and $\mathrm{BC}$ without $\mathrm{BM}$ in non-triple negative subtype IDC patients $(P=0.026)$. E. Comparison of BMPRIB expression in $\mathrm{BC}$ with $\mathrm{BM}$ and $\mathrm{BC}$ without $\mathrm{BM}$ in luminal subtype IDC patients $(P=0.027)$. F. BC patients with low expression of BMPRIB exhibited earlier occurrence of BM. The median interval time from diagnosis of $\mathrm{BC}$ to $\mathrm{BM}$ in patients with low expression of BMPRIB was shorter than the high BMPRIB expression group (MannWhitney $\mathrm{U}$ test, $P=0.011)$. G. BC patients with low expression of BMPRIB exhibited shorter survival after diagnosis of BM. The median interval time from diagnosis of BM to the follow-up ending time of patients with low expression of BMPRIB was shorter than that of high BMPRIB expression patients $(P=0.016)$. (B-G: Mann-Whitney U test). 
A
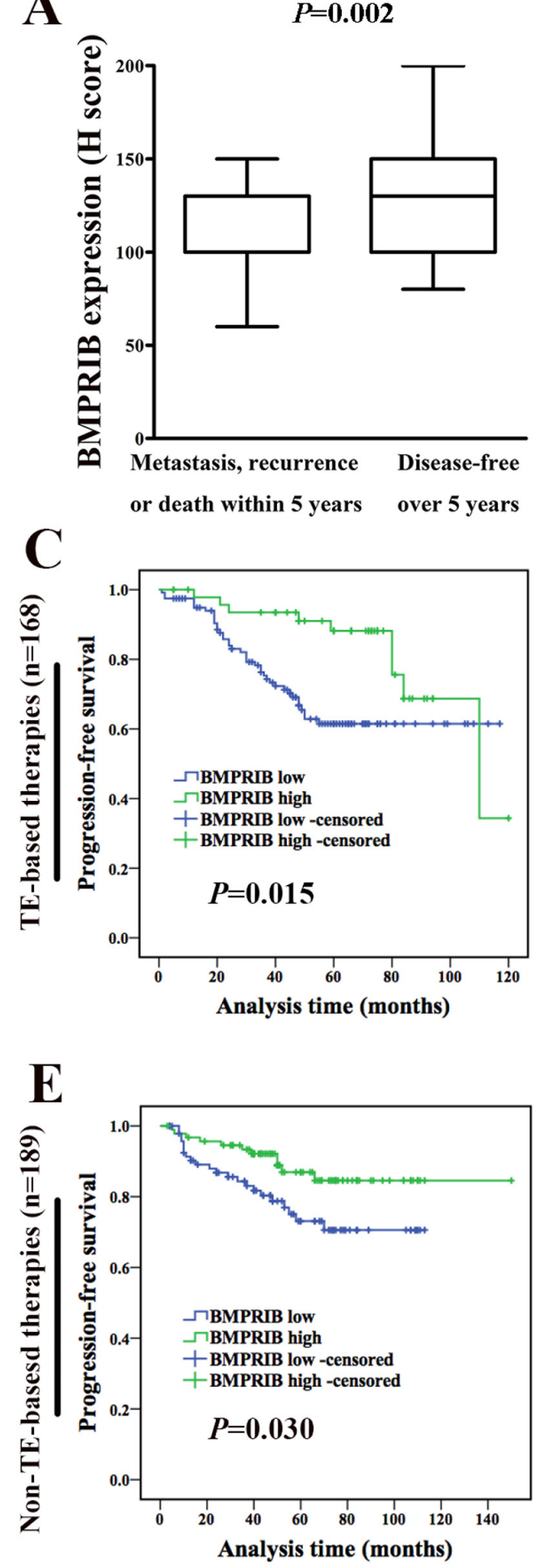

B

$P<0.001$

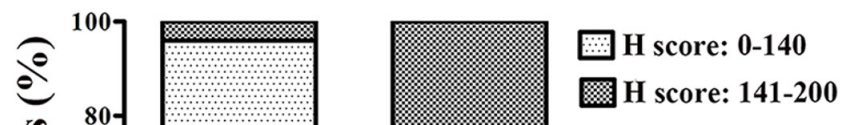

D

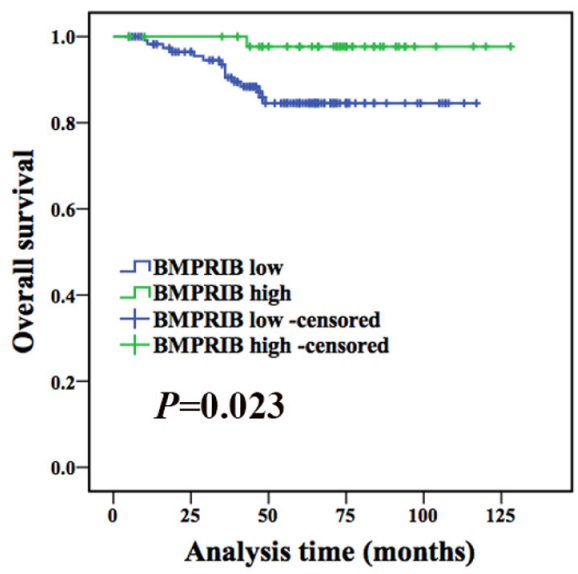

$\mathbf{F}$

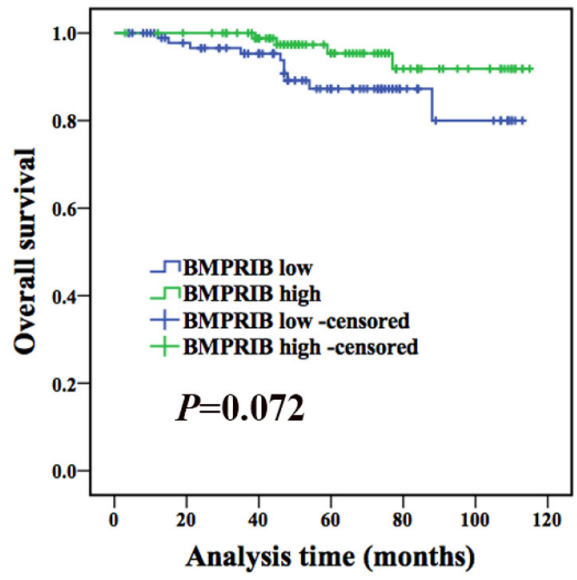

Figure 5: High BMPRIB expression indicated favorable prognosis in breast cancer patients treated with TE-based therapies. A. Among 168 TE-based therapies, BMPRIB expression in patients who developed metastasis, recurrence or death within 5 years was lower than that in those patients who were disease-free over 5 years (Mann-Whitney $\mathrm{U}$ test, $P=0.002$ ). B. Percentage of cases with low and high expression of BMPRIB with regard to patients treated with TE-based therapies' prognosis. 96.6\% (28/29) of patients that developed metastasis, recurrence or death within 5 years showed lower BMPRIB expression compared with 59.7\% (40/67) patients who were disease-free over 5 years $\left(\chi^{2}\right.$ test, $\left.P<0.001\right)$. C and $\mathbf{D}$. Progression-free survival (PFS) and overall survival (OS) curves of IDC patients treated with TE-based therapies $(\mathrm{n}=168)$ with BMPRIB expression, respectively (log-rank test). $\mathbf{E}$ and $\mathbf{F}$. PFS and OS curves of IDC patients treated with non-TE-based therapies $(n=189)$ with BMPRIB expression, respectively. 


\section{A $1 \begin{gathered}\text { Core needle biopsy specimens of } \\ \text { pre-neoadjuvant chemotherapy }\end{gathered}$}

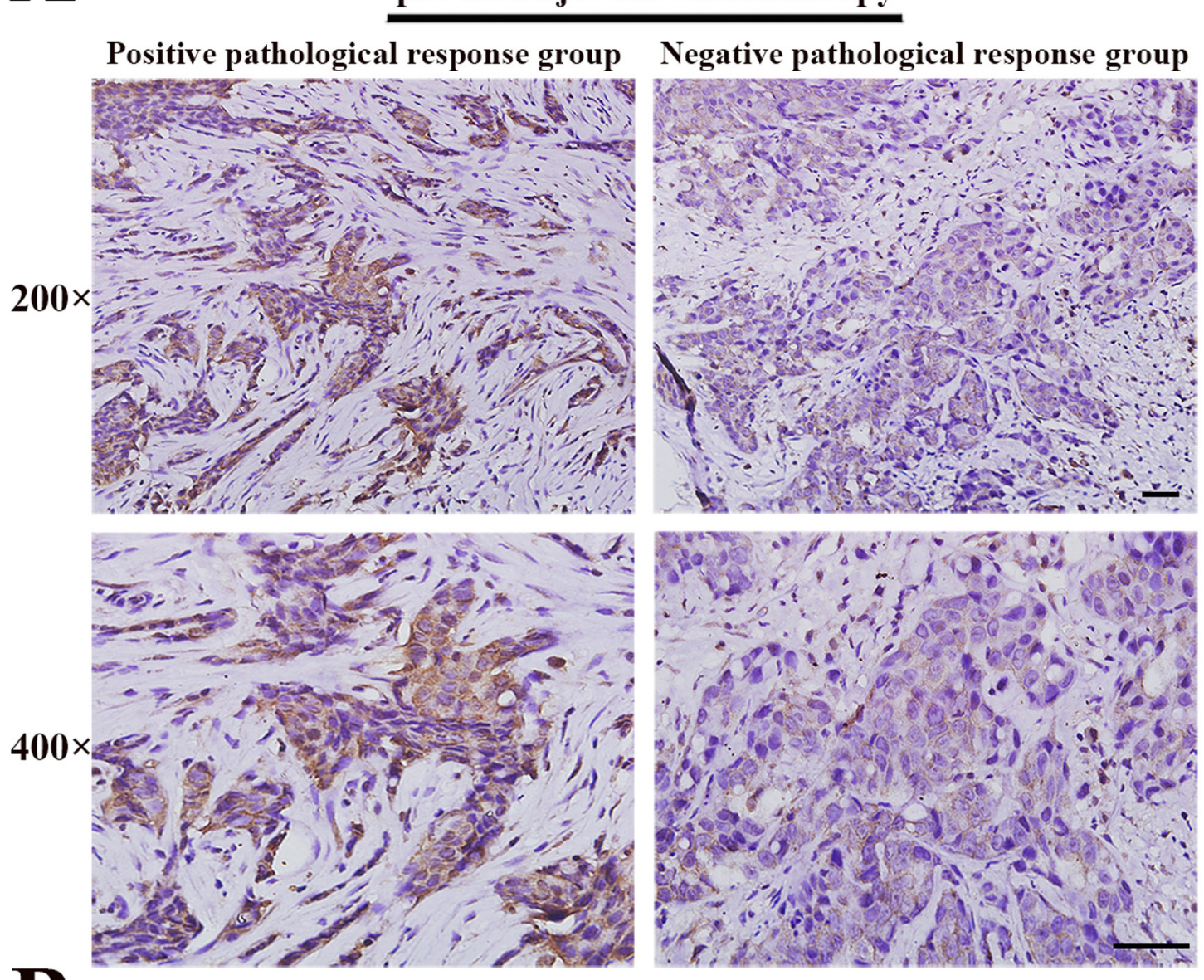

B

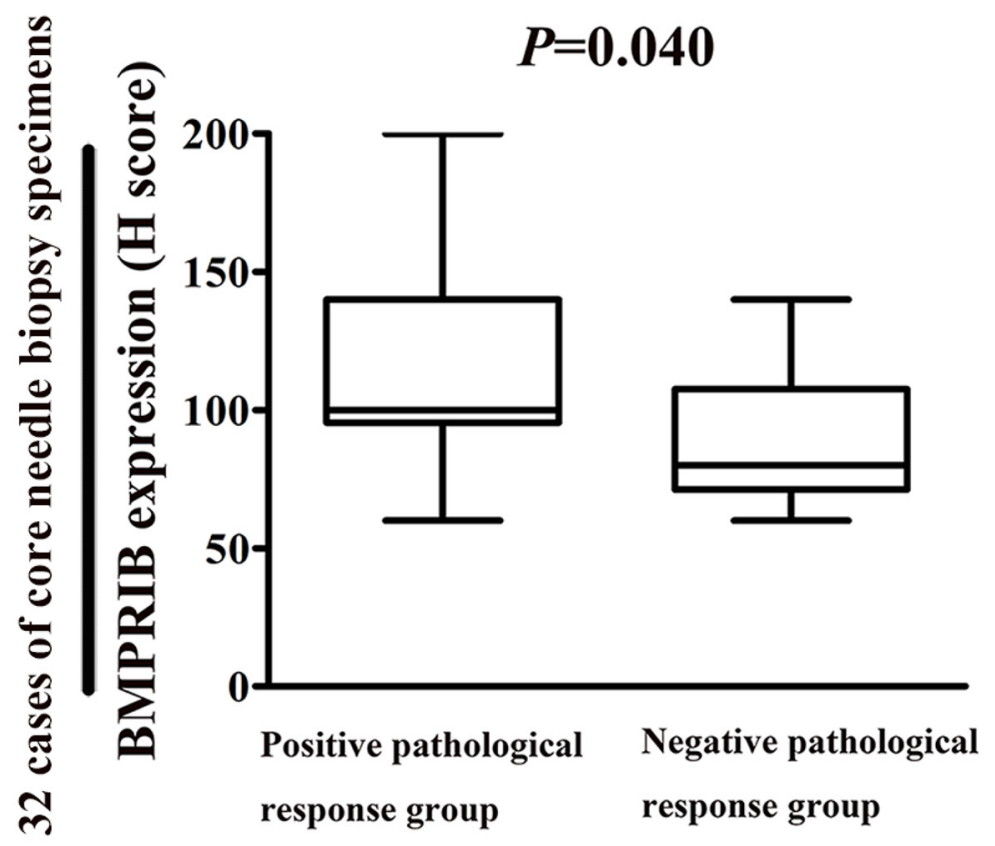

Figure 6: High BMPRIB expression was sensitive to TE therapy by using 32 specimens of core needle biopsy before neoadjuvant chemotherapy. A. Representative immunohistochemical images of BMPRIB expression in both positive and negative pathological response groups (magnification $200 \times$ or $400 \times$ ). B. BMPRIB expression was higher in positive pathological response group than negative pathological response group (Mann-Whitney $\mathrm{U}$ test, $P=0.040) .(\mathrm{Bar}=100 \mu \mathrm{m})$ 
A

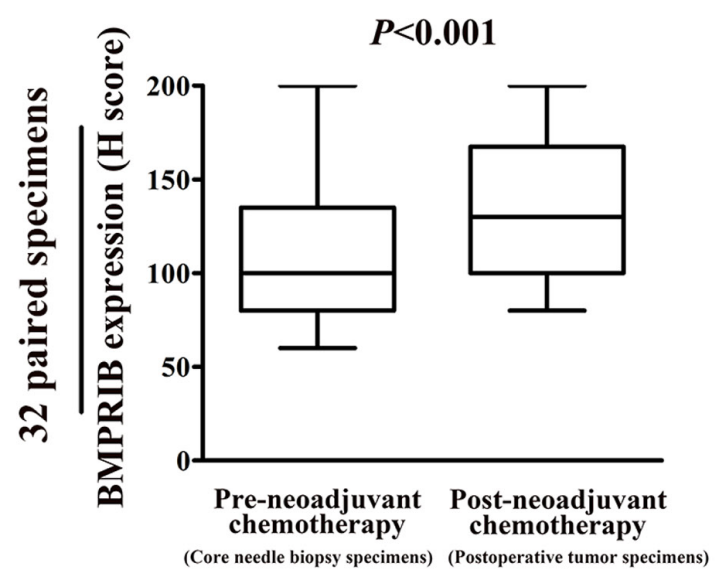

B

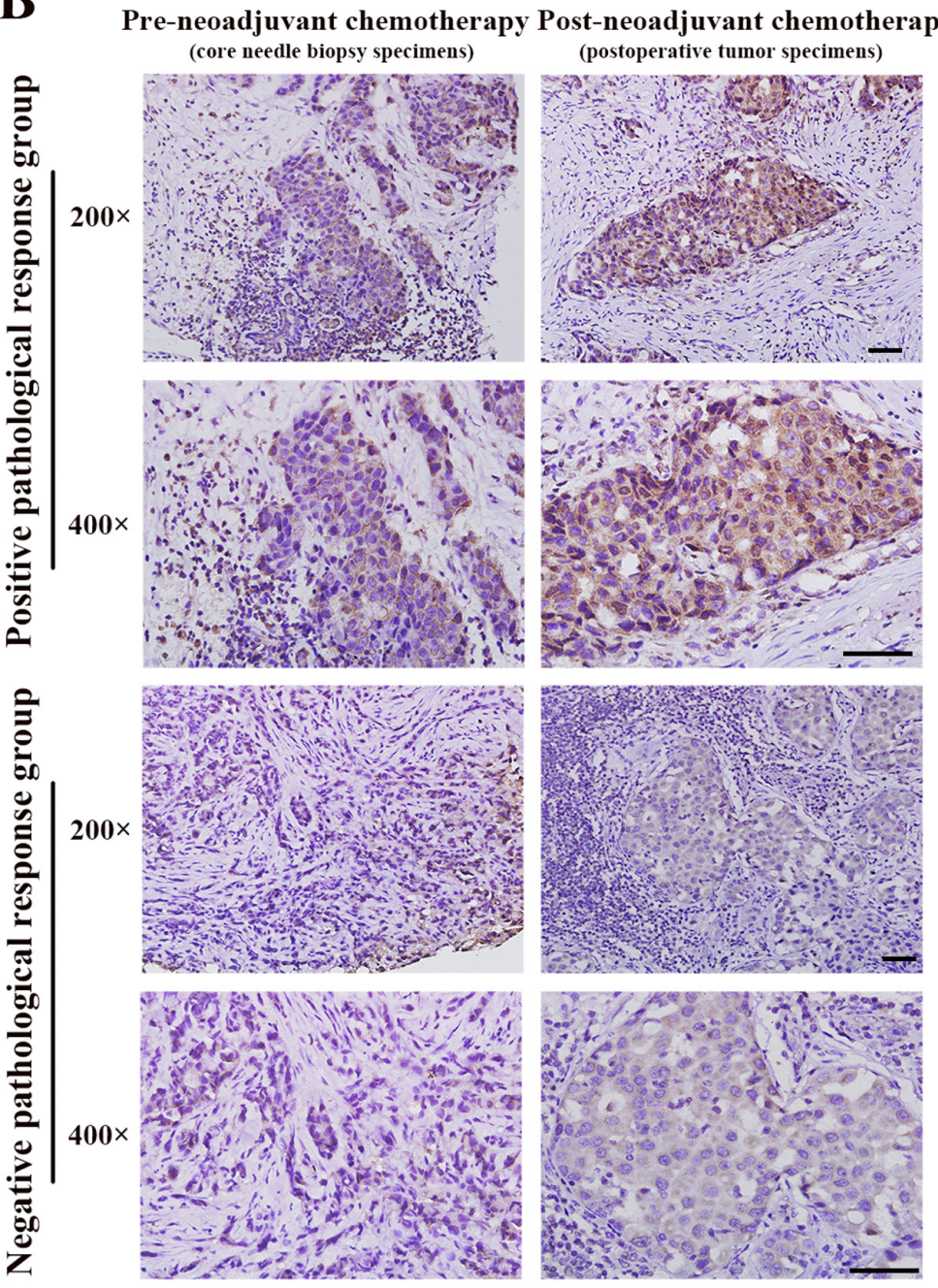

Figure 7: Expression of BMPRIB was up-regulated after TE neoadjuvant chemotheapy, which was mainly contributed by the positive pathological response group. A. Expression of BMPRIB was up-regulated after TE neoadjuvant chemotheapy by 32 paired specimens of pre- and post-neoadjuvant chemotherapy (Mann-Whitney $\mathrm{U}$ test, $P<0.001$ ). B. In positive pathological response group, BMPRIB expression was up-regulated in postoperative tumor specimens compared with their paired core needle biopsy specimens. Representative immunohistochemical images of BMPRIB expression in positive pathological response group were shown in upper part (magnification $200 \times$ or $400 \times$ ). In negative pathological response group, there was not an obvious up-regulated BMPRIB expression in the postoperative tumor specimens compared with their paired core needle biopsy specimens. Representative immunohistochemical images of BMPRIB expression in negative pathological response group were shown in lower part (magnification $200 \times$ or $400 \times)$. $(\mathrm{Bar}=100 \mu \mathrm{m})$ 
Table 4: Relationship between BMPRIB expression and TE neo-adjuvant chemotherapy in 32 paired specimens

\begin{tabular}{|c|c|c|c|}
\hline \multicolumn{3}{|c}{ Low (0-140) } & \multicolumn{1}{c}{ High (141-200) } \\
\hline Total & & & $0.007^{*}$ \\
\hline Pre-neoadjuvant chemotherapy & $28(87.5)$ & $4(12.5)$ & \\
\hline Post-neoadjuvant chemotherapy & $19(59.4)$ & $13(40.6)$ & \\
\hline Positive pathological response & & & $0.034^{*}$ \\
\hline Pre-neoadjuvant chemotherapy & $16(80.0)$ & $4(20.0)$ & \\
\hline Post-neoadjuvant chemotherapy & $10(50.0)$ & $10(50.0)$ & \\
\hline Negative pathological response & & & 0.083 \\
\hline Pre-neoadjuvant chemotherapy & $12(100.0)$ & $0(0.0)$ & \\
\hline Post-neoadjuvant chemotherapy & $9(75.0)$ & $3(25.0)$ & \\
\hline
\end{tabular}

Statistical analysis was used Wilcoxon matched pairs signed rank test.

Table 4, Figure 7B). In negative pathological response group, there was not an obvious increase of BMPRIB expression in the post-neoadjuvant chemotherapy specimens compared with their paired pre-neoadjuvant chemotherapy specimens $(P=0.083$, Table 4, Figure 7B).

\section{DISCUSSION}

Multiple factors were reported to participate in breast cancer bone metastasis [17-23]. Buijs et al. showed that high expression of BMP 7 (a member of BMP family) inhibited the formation of bone metastases in breast cancer patients [24]. Lewis et al. found loss of BMPRIB induced increased expression of cytokeratin17, which is a promoting factor of invasion [6]. In our present study, novel evidence is provided that low level of BMPRIB was a promoting factor for breast cancer patients to develop bone metastasis, but not lung, liver or brain.

TE-based therapies are part of the standard of care in first line treatment of metastatic breast cancer and their clinical use is widespread [25-26]. Actually, only about $15 \%$ patients could achieve pathologic complete response, thus a more detailed classification is necessary to screen a more suitable population to TE regimens [27]. A potential predictor of response to TE chemotherapy is urgently needed.

We reported for the first time that high BMPRIB expression was sensitive to TE-based chemotherapy by two cohorts of population. The survival analysis revealed that low expression of BMPRIB indicated a poor prognosis in 168 TE-based patients. In addition, by 32 paired preand post-neoadjuvant chemotherapy specimens, patients with low BMPRIB expression were insensitive to TE neoadjuvant chemotherapy. Dzietczenia et al. found that leukemia patients with a complete or partial response to anthracyclines-based chemotherapy exhibited higher expression of BMPRIB than those patients without a response to treatment which was consistent with our findings in breast cancer [28].

Overall, our study concluded that low expression of BMPRIB indicated poor prognosis of breast cancer and was insensitive to taxane-anthracycline chemotherapy. Our findings also lay a foundation to help clinicians improve identification of patients for TE regimens by BMPRIB in the era of precision medicine.

\section{MATERIALS AND METHODS}

\section{Patient selection and clinical information}

Paraffin-embedded specimens of 368 breast cancer patients with invasive ductal carcinoma (IDC), diagnosed between 2004 and 2009, together with 40 cases of breast ductal carcinoma in situ (DCIS) and 52 cases of nonneoplastic tissues were reviewed and randomly selected from the archives of the Department of Breast Cancer Pathology and Research Laboratory, Tianjin Medical University Cancer Institute \& Hospital (Tianjin, China) for this study. The histopathology was reviewed and diagnosis in each case was confirmed independently by two pathologists according to World Health Organization (WHO) criteria. This study was reviewed and approved by the Institutional Ethic Committee of Tianjin Medical University Cancer Institute \& Hospital. All the patients signed an informed consent for participation of the study and the use of their biological tissues.

368 IDC patients were women aging from 28 to 89 years (mean 51.5 years) without preoperative chemotherapy or radiation. A total of 357 cases were included for prognostic analyses, excluding cases with no follow-up data (11 cases). These patients were followed up with a median of 59 months (3-180 months). Recurrences 
and distant metastasis were recorded for $20(5.4 \%)$ cases and $51(13.9 \%)$ cases respectively, and $32(8.7 \%)$ patients died. Among 357 cases, 278 (77.9\%) patients were luminal subtype, $23(6.4 \%)$ patients were HER2-overexpression subtype and $56(15.7 \%)$ were triple negative subtype.

$168(47.1 \%)$ patients received TE (taxane + anthracycline) based chemotherapies, the rest (189 cases, 52.9\%) were treated with other chemotherapies (not TE-based therapies) after operation. The details of patients who received non-TE based chemotherapy were in the following: 86 cases (CEF/CAF); 76 cases (CMF); 7 cases (Platinum-based chemotherapy); 4 cases (Taxane); 16 cases (unknown). (CEF: Cyclophosphamide, epirubicin and 5-fluorouracil; CAF: Cyclophosphamide, doxorubicin and 5-fluorouracil; CMF: Cyclophosphamide, methotrexate and 5-fluorouracil)

\section{Patients' prognostic information}

Among 357 patients with prognostic analyses, there were 48 developed metastases, recurrence or death within 5 years; while 144 patients were disease-free over 5 years since diagnosis of breast cancer. Among patients who received TE-based regimens, 29 patients developed metastasis, recurrence or death within 5 years; while 67 patients who were disease-free over 5 years.

Among 357 patients with prognostic analyses, there were 51 who developed distant metastasis during the follow-up period, 39 patients developed bone metastasis, 12 patients developed lung metastasis, 13 patients developed liver metastasis, 5 patients developed brain metastasis, 2 patients developed uterus metastasis and 3 additional cases developed kidney, ovarian and thyroid metastasis, respectively. It was worth noting that multiple organic metastases were noted in 14 patients. Among those 39 IDC patients with bone metastasis, 30 patients were luminal subtype, 1 patient was HER2-overexpression subtype and 8 were triple-negative subtype.

\section{Information of 32 paired specimens from pre-neoadjuvant and post-neoadjuvant chemotherapy}

We also randomly selected another cohort of patients (32 cases) hospitalized during October 2005 to June 2009. All 32 patients were diagnosed with invasive breast cancer by 14-gauge core needle biopsy and then had completed with preoperative neoadjuvant chemotherapy consisted of 2 to 8 cycles of TE combined chemotherapy regimen without other local or systemic treatment before surgery. Patients were women 28 to 71 years of age (mean age 52.6 years) and had no other malignant tumors or tumor history. The distribution of clinical involvement showed that all the patients had tumors $>2.0 \mathrm{~cm}$. These 32 paired specimens were collected from each patient's core needle biopsy specimens of primary breast tumor before neoadjuvant chemotherapy and its matching postoperative tumor tissues. All specimens were immediately fixed in $10 \%$ normal-buffered formalin and embedded in paraffin and stained for the presence of BMPRIB by immunohistochemistry.

The pathological response to neoadjuvant chemotherapy was evaluated after surgical resection of the remaining tumor and assessed according to Miller and Payne histological grading system: grade 1, no change or some alteration to individual malignant cells but no reduction in overall cellularity; grade 2, minor loss (up to $30 \%$ ) of cancer cells but overall cellularity remains high; grade 3 , reduction of $30 \%$ to $90 \%$ of cancer cells; grade 4 , more than $90 \%$ loss of cancer cells but small clusters or widely dispersed individual cancer cells remain; grade 5, no malignant cells identifiable in sections from the site of the tumor consisting of vascular fibroblastic stroma, often containing macrophages; however, ductal carcinoma in situ (DCIS) may be present [29]. Of these 32 patients, there were 12 grade 1 responses, 9 grade 2 responses, 7 grade 3 responses and 4 grade 4 responses, but no grade 5 responses. In this study, the 32 patients were divided into two groups: one group was pathological response grade 2 to 4 which was regarded as positive and another group was pathological response grade 1 which was regarded as negative.

\section{Immunohistochemical staining}

BMPRIB expression was examined by histochemistry techniques and S-P method. In brief, Sections ( $5 \mu \mathrm{m}$ thick) were dewaxed, hydrated, and heated for $2.5 \mathrm{~min}$ for antigen retrieval in a conventional pressure cooker by using citrate buffer, $\mathrm{pH}$ 6.0. Then sections were treated with $3 \% \mathrm{H}_{2} \mathrm{O}_{2}$ for 20 min to reduce endogenous activity and incubated with $10 \%$ normal goat serum for 20 min to eliminate nonspecific staining. Next, the primary antibody against BMPRIB (rabbit anti-human polyclonal antibody, Santa Cruz, USA, sc-25455, 1:100) was applied at $4^{\circ} \mathrm{C}$ overnight. After washing, biotin labeled secondary antibody against rabbit immunoglobulin was applied for $20 \mathrm{~min}$ at room temperature. The slides were rinsed and covered with streptavidin-biotin-peroxidase for $20 \mathrm{~min}$. All sections were stained with 3,3'-diaminobenzidinetetrahydrochloride (DAB). Slides were counterstained with hematoxylin and mounted for light microscopy.

\section{Evaluation of staining}

Using light microscopy, stained tissue sections were reviewed by two pathologists in a blinded manner. A consensus judgment was adopted for the intensity score of the tumors based on the strength of BMPRIB expression. 0 (-): no or low staining; $1(+)$ : moderate staining; $2(++)$ : strong staining. Percentage of the positive staining was scored as 0-100. An immunohistochemical score (H score) was ranged from 0 to 200 by multiplying the intensity and the percentage score. Patients were categorized into 
groups according to $\mathrm{H}$ score of BMPRIB: low BMPRIB expression (0-140), high BMPRIB expression (141-200).

Immunohistochemistry for estrogen receptor (ER) and progesterone receptor (PR) was re-evaluated using the 2010 American Society of Clinical Oncology/ College of American Pathologists (ASCO/CAP) guideline. Cases were scored positive for ER and PR if nuclear immunoreactivity was present in more than $1 \%$ of tumor cells [30]. Immunohistochemistry for human epidermal growth factor receptor 2 (HER2) was re-evaluated using the $2014 \mathrm{ASCO} / \mathrm{CAP}$ updated guideline [31].

\section{Western blot}

Western blot analysis was performed to evaluate the expression of BMPRIB in both non-neoplastic breast tissues adjacent to tumor and breast tumor tissues. Frozen breast tumor specimens (13 cases) and non-neoplastic breast tissues adjacent to tumor (13 cases) were collected between 2012 and 2015. All patients were women without preoperative chemotherapy or radiation. Tissues were lysed in $1 \times$ SDS lysis buffer (Tris-HCl, $\mathrm{pH} 6.8,62.5 \mathrm{mM}$, $2 \%$ SDS, $10 \%$ glycerol) for 60 minutes on ice directly. Equal amounts of protein were separated by SDS-PAGE, and electrotransferred onto nitrocellulose membranes. Blots were analyzed by LiCor Odyssey infrared imaging.

\section{Statistical methods}

The SPSS 13.0 software package (SPSS, Chicago, IL, USA) was used for statistical analysis. Mann-Whitney $\mathrm{U}$ test and $\chi^{2}$ test were performed for group comparisons and correlations between two variables were evaluated by Spearman rank correlation test. The relation between BMPRIB expression and distant metastasis was evaluated using Spearman rank correlation test. Progression-free survival (PFS) was defined as the time from surgery to recurrence or cancer-specific death, whichever occurred first. Overall survival (OS) was calculated from pathological diagnosis to the date of last contact or death from breast carcinoma. Survival analyses were performed according to the Kaplan-Meier method. Wilcoxon matched pairs signed rank test was performed for analyzing the expression of BMPRIB in 32 paired specimens. All statistical tests were 2-tailed and $P<0.05$ was regarded as significant.

\section{CONFLICTS OF INTEREST}

The authors declare no conflicts of interest.

\section{GRANT SUPPORT}

This work was supported by China 863 program (2012AA020101) and National Scientific Foundation of China (81272358) from Dr. Feng Gu; National Scientific
Foundation of China (81572851) from Dr. Yongjie Ma and National Scientific Foundation of China (31471331) from Dr. Caixia Guo.

\section{REFERENCES}

1. Siegel R, Naishadham D, Jemal A. Cancer statistics, 2013. CA Cancer J Clin. 2013; 63:11-30.

2. Yoneda T, Hiraga T. Crosstalk between cancer cells and bone microenvironment in bone metastasis. Biochem Biophys Res Commun. 2005; 328:679-687.

3. Plunkett TA, Smith P, Rubens RD. Risk of complications from bone metastases in breast cancer. implications for management. Eur J Cancer. 2000; 36:476-482.

4. Guo X, Wang XF. Signaling cross-talk between TGF-beta/ BMP and other pathways. Cell Res. 2009; 19:71-88.

5. Bokobza SM, Ye L, Kynaston HE, Mansel RE, Jiang WG. Reduced expression of BMPR-IB correlates with poor prognosis and increased proliferation of breast cancer cells. Cancer Genomics Proteomics. 2009; 6:101-108.

6. Lewis CJ, Mardaryev AN, Poterlowicz K, Sharova TY, Aziz A, Sharpe DT, Botchkareva NV, Sharov AA. Bone morphogenetic protein signaling suppresses wound-induced skin repair by inhibiting keratinocyte proliferation and migration. J Invest Dermatol. 2014; 134:827-837.

7. Ye L, Kynaston H, Jiang WG. Bone morphogenetic protein-9 induces apoptosis in prostate cancer cells, the role of prostate apoptosis response-4. Mol Cancer Res. 2008; 6:1594-1606.

8. Ide H, Katoh M, Sasaki H, Yoshida T, Aoki K, Nawa Y, Osada Y, Sugimura T, Terada M. Cloning of human bone morphogenetic protein type IB receptor (BMPR-IB) and its expression in prostate cancer in comparison with other BMPRs. Oncogene. 1997; 14:1377-1382.

9. Kim IY, Lee DH, Ahn HJ, Tokunaga H, Song W, Devereaux LM, Jin D, Sampath TK, Morton RA. Expression of bone morphogenetic protein receptors type-IA, -IB and -II correlates with tumor grade in human prostate cancer tissues. Cancer Res. 2000; 60:2840-2844.

10. Liu S, Yin F, Fan W, Wang S, Guo XR, Zhang JN, Tian ZM, Fan M. Over-expression of BMPR-IB reduces the malignancy of glioblastoma cells by upregulation of p21 and p27Kip1. J Exp Clin Cancer Res. 2012; 31:52.

11. Ma Y, Ma L, Guo Q, Zhang S. Expression of bone morphogenetic protein-2 and its receptors in epithelial ovarian cancer and their influence on the prognosis of ovarian cancer patients. J Exp Clin Cancer Res. 2010; 29:85.

12. Komai Y, Morimoto S, Saito K, Urushibara M, Sakai K, Ikeda S. Possible involvement of bone morphogenetic protein 2 in heterotopic ossification in metastatic lesion from urothelial carcinoma of bladder. Int J Urol. 2006; 13:1126-1128.

13. Helms MW, Packeisen J, August C, Schittek B, Boecker W, Brandt BH, Buerger H. First evidence supporting a 
potential role for the BMP/SMAD pathway in the progression of oestrogen receptor-positive breast cancer. J Pathol. 2005; 206:366-376.

14. Tanaka T, Bai T, Yukawa K, Umesaki N. Optimal combination chemotherapy and chemoradiotherapy with etoposide for advanced cervical squamous cancer cells in vitro. Oncol Rep. 2006; 15:939-947.

15. Lu H, Murtagh J, Schwartz EL. The microtubule binding drug laulimalide inhibits vascular endothelial growth factorinduced human endothelial cell migration and is synergistic when combined with docetaxel (taxotere). Mol Pharmacol. 2006; 69:1207-1215.

16. North HA, Pan L, McGuire TL, Brooker S, Kessler JA. beta1-Integrin alters ependymal stem cell BMP receptor localization and attenuates astrogliosis after spinal cord injury. J Neurosci. 2015; 35:3725-3733.

17. Buijs JT, van der Pluijm G. Osteotropic cancers: from primary tumor to bone. Cancer Lett. 2009; 273:177-193.

18. Miwa S, Yano S, Zhang Y, Matsumoto Y, Uehara F, Yamamoto M, Hiroshima Y, Kimura H, Hayashi K, Yamamoto N, Bouvet M, Tsuchiya H, Hoffman RM, et al. Tumor-targeting Salmonella typhimurium A1-R prevents experimental human breast cancer bone metastasis in nude mice. Oncotarget. 2014; 5:7119-7125. doi:10.18632/ oncotarget.2226.

19. Choi B, Lee EJ, Song DH, Yoon SC, Chung YH, Jang Y, Kim SM, Song Y, Kang SW, Yoon SY, Chang EJ. Elevated Pentraxin 3 in bone metastatic breast cancer is correlated with osteolytic function. Oncotarget. 2014; 5:481-492. doi:10.18632/oncotarget.1664.

20. Miwa S, Yano S, Zhang Y, Matsumoto Y, Uehara F, Yamamoto M, Hiroshima Y, Kimura H, Hayashi K, Yamamoto N, Bouvet M, Tsuchiya H, Hoffman RM, et al. Tumor-targeting Salmonella typhimurium A1-R prevents experimental human breast cancer bone metastasis in nude mice. Oncotarget. 2014; 5:7119-7125. doi:10.18632/ oncotarget.1664.

21. Kovacheva M, Zepp M, Berger SM, Berger MR. Sustained conditional knockdown reveals intracellular bone sialoprotein as essential for breast cancer skeletal metastasis. Oncotarget. 2014; 5:5510-5522. doi: 10.18632/oncotarget.2132.

22. Tang X, Mahajan SS, Nguyen LT, Beliveau F, Leduc R, Simon JA, Vasioukhin V. Targeted inhibition of cellsurface serine protease Hepsin blocks prostate cancer bone metastasis. Oncotarget. 2014; 5:1352-1362. doi:10.18632/ oncotarget.1817.

23. Choi B, Lee EJ, Song DH, Yoon SC, Chung YH, Jang Y, Kim SM, Song Y, Kang SW, Yoon SY, Chang EJ. Elevated Pentraxin 3 in bone metastatic breast cancer is correlated with osteolytic function. Oncotarget. 2014; 5:481-492. doi:10.18632/oncotarget.1664.

24. Buijs JT, Henriquez NV, van Overveld PG, van der Horst G, Que I, Schwaninger R, Rentsch C, Ten Dijke P, CletonJansen AM, Driouch K, Lidereau R, Bachelier R, Vukicevic S, Clezardin P, Papapoulos SE, Cecchini MG, et al. Bone morphogenetic protein 7 in the development and treatment of bone metastases from breast cancer. Cancer Res. 2007; 67:8742-8751.

25. Giordano SH, Buzdar AU, Smith TL, Kau SW, Yang Y, Hortobagyi GN. Is breast cancer survival improving? Cancer. 2004; 100:44-52.

26. Crown J, Dieras V, Kaufmann M, von Minckwitz G, Kaye S, Leonard R, Marty M, Misset JL, Osterwalder B, Piccart M. Chemotherapy for metastatic breast cancer-report of a European expert panel. Lancet Oncol. 2002; 3:719-727.

27. von Minckwitz G, Untch M, Blohmer JU, Costa SD, Eidtmann H, Fasching PA, Gerber B, Eiermann W, Hilfrich J, Huober J, Jackisch C, Kaufmann M, Konecny GE, et al. Definition and impact of pathologic complete response on prognosis after neoadjuvant chemotherapy in various intrinsic breast cancer subtypes. J Clin Oncol. 2012; 30:1796-1804.

28. Dzietczenia J, Wrobel T, Jazwiec B, Mazur G, Butrym A, Kuliczkowski K. Expression of cyclin A and bone morphogenetic protein receptors and response to induction therapy in patients with acute leukemias. Leuk Lymphoma. 2011; 52:2336-2341

29. Ogston KN, Miller ID, Payne S, Hutcheon AW, Sarkar TK, Smith I, Schofield A, Heys SD. A new histological grading system to assess response of breast cancers to primary chemotherapy: prognostic significance and survival. Breast. 2003; 12:320-327.

30. Hammond ME, Hayes DF, Dowsett M, Allred DC, Hagerty KL, Badve S, Fitzgibbons PL, Francis G, Goldstein NS, Hayes M, Hicks DG, Lester S, Love R, et al. American Society of Clinical Oncology/College of American Pathologists guideline recommendations for immunohistochemical testing of estrogen and progesterone receptors in breast cancer. Arch Pathol Lab Med. 2010; 134:907-922.

31. Wolff AC, Hammond ME, Hicks DG, Dowsett M, McShane LM, Allison KH, Allred DC, Bartlett JM, Bilous M, Fitzgibbons P, Hanna W, Jenkins RB, Mangu PB, et al. Recommendations for human epidermal growth factor receptor 2 testing in breast cancer: American Society of Clinical Oncology/College of American Pathologists clinical practice guideline update. Arch Pathol Lab Med. 2014; 138:241-256. 